\title{
BMJ Open Defining high probability when making a diagnosis of asthma in primary care: mixed-methods consensus workshop
}

\author{
Luke Daines (D) , ${ }^{1}$ Steff Lewis, ${ }^{1}$ Antonius Schneider, ${ }^{2}$ Aziz Sheikh, ${ }^{1}$ Hilary Pinnock ${ }^{1}$
}

To cite: Daines L, Lewis S, Schneider A, et al. Defining high probability when making a diagnosis of asthma in primary care: mixed-methods consensus workshop. BMJ Open 2020;10:e034559. doi:10.1136/ bmjopen-2019-034559

- Prepublication history for this paper is available online. To view these files, please visit the journal online (http://dx.doi org/10.1136/bmjopen-2019034559).

Received 25 September 2019 Revised 04 March 2020 Accepted 01 April 2020

Check for updates

(c) Author(s) (or their employer(s)) 2020. Re-use permitted under CC BY-NC. No commercial re-use. See rights and permissions. Published by BMJ.

${ }^{1}$ Asthma UK Centre for Applied Research, Usher Institute, The University of Edinburgh, Edinburgh, UK

${ }^{2}$ TUM School of Medicine, Institute of General Practice and Health Services Research, Technical University of Munich, Munich, Germany

Correspondence to

Dr Luke Daines;

luke.daines@ed.ac.uk

\section{ABSTRACT}

Objective Making the diagnosis of asthma is challenging. Guidelines recommend that clinicians identify a group at 'high probability' of asthma. High probability, however, is not numerically defined giving rise to uncertainty. The aim of this work was to build consensus on what constitutes a high probability of asthma in primary care. High probability was defined as the probability threshold at which there is enough information to make a firm diagnosis of asthma, and a subsequent negative test would not alter that opinion (assumed to be a false negative).

Design Mixed-methods study.

Setting A consensus workshop using modified nominal group technique was held during an international respiratory conference.

Participants International conference attendees eligible if they had knowledge/experience of working in primary care, respiratory medicine and spoke English.

Methods Participants took part in facilitated discussions and voted over three rounds on what constituted a high probability of asthma diagnosis. The workshop was audiorecorded, transcribed and qualitatively analysed.

Results Based on final votes, the mean value for a high probability of asthma in primary care was $75 \%$ (SD 7.6), representing a perceived trade-off between limiting the number of false positives (more likely if a lower threshold was used) and pragmatism on the basis that first-line preventive therapies (ie, low-dose inhaled corticosteroids) are relatively low risk. The need to review response to treatment was strongly emphasised for detecting nonresponders and reviewing the diagnosis.

Conclusion A consensus probability of $75 \%$ was the threshold at which the primary care participants in this workshop felt confident to establish the diagnosis of asthma, albeit with the caveat that a review of treatment response was essential. Contextual factors, including availability and timing of tests and the ease with which patients could be reviewed, influenced participants' decision making.

\section{BACKGROUND}

Misdiagnosis of asthma is common with both underdiagnosis and overdiagnosis reported. ${ }^{1-3}$ Asthma is difficult to diagnose because it is a heterogeneous disease with different underlying disease processes, defined by variable symptoms and expiratory airflow limitation. ${ }^{4}$ Investigations can determine key features of

\section{Strengths and limitations of this study}

This study achieved consensus on a value for 'high probability' of asthma diagnosis in primary care.

- Qualitative analysis provided insight into the contextual factors that affect the decision making of health professionals when assessing someone with symptoms to suggest asthma.

- We recruited 10 participants, the suggested number for a modified nominal group technique workshop, yet because of the small sample, the statistical analysis should be interpreted cautiously and in conjunction with the qualitative analysis.

- Participants were recruited from an international conference and, reflecting the majority of delegates, were doctors working in primary care from around the world with interest in respiratory medicine.

- We chose not to include clinicians from secondary/tertiary care because health problems typically present differently between primary and secondary care settings.

asthma, but all have limitations. For instance, spirometry is considered the reference standard for diagnosing airway obstruction, ${ }^{5}$ but airway obstruction may not be persistent or present in all cases of asthma. ${ }^{6}$ Bronchial provocation is the reference standard for determining bronchial hyper-responsiveness ${ }^{7}$ but is time-consuming, carries a risk of inducing severe bronchospasm ${ }^{8}$ and is not widely available internationally. Fractional exhaled nitric oxide (FeNO) identifies airway inflammation and is useful for ruling in but less helpful for ruling out asthma. ${ }^{9}$ Peak flow variability is straightforward to perform but has low diagnostic accuracy for asthma. ${ }^{10}{ }^{11} \mathrm{In}$ the absence of an investigation that can rule in or rule out asthma with high certainty, the diagnosis of asthma is often made clinically.

Therefore, if asthma is suspected in people presenting with undifferentiated symptoms (typically to primary care), health professionals are recommended to build up evidence for or against the presence of the diagnosis by undertaking a structured clinical 
assessment. ${ }^{41213}$ Current guidelines differ in their recommendations about the steps needed to achieve an asthma diagnosis. ${ }^{41213}$ The Global Initiative for Asthma and the National Institute for Health and Care Excellence advise that anyone suspected of asthma should be assessed with spirometry, peak flow variability and/or FeNO. ${ }^{413}$ If uncertainty remains after initial investigations, repeating tests after an interval or additional testing (such as bronchial provocation) is advised. ${ }^{4} 13$ The British Thoracic Society (BTS)/Scottish Intercollegiate Guideline Network (SIGN) advise weighing up the probability that an individual has asthma. ${ }^{12}$ In the case of intermediate or low probability, further investigations are necessary. ${ }^{12}$ Individuals judged to be at high probability of asthma may be coded as having suspected asthma and a monitored trial of treatment initiated with clear plans for reassessment. ${ }^{12}$

The differences between asthma guidelines have unfortunately led to confusion among some health professionals. ${ }^{14}$ Furthermore, as asthma is a variable, heterogeneous condition, the clinical picture is not always obvious, and in some instances, diagnostic investigations, which may deliver false-positive or false-negative results, can add to uncertainty.

The probability of asthma is informed by all available information, which will include a clinical assessment, previous clinical records, as well as the results of any investigations that have been undertaken. Choosing if and when to conduct a further investigation or to commence treatment is conceptualised in the threshold approach to diagnosis. ${ }^{15}$ The 'test-treatment' threshold indicates the point at which further testing would be unlikely to alter the probability of the diagnosis. If an individual is below the test-treatment threshold, then further information or investigations are likely to help decision making and should be completed. ${ }^{15}$ If the probability is higher than the test-treatment threshold, further investigations are unlikely to improve the probability, so the diagnosis can be confirmed and treatment started. ${ }^{15}$ In the context of asthma, probability above the test-treatment threshold would lead to a 'suspected asthma' code and a monitored trial of treatment. ${ }^{12}$

Having a numerically defined threshold could bring clarity over what guidelines describe as 'high probability' of asthma, ${ }^{12}$ which would also facilitate greater consistency between clinicians regardless of their levels of experience. Second, in the context of clinical prediction models for asthma diagnosis, ${ }^{16}$ it may be possible to input clinical assessment and investigation results into an algorithm and to generate a numerical probability that asthma is present. A consensus on the test-treatment threshold could help clinicians to make sense of the output of such algorithms.

Therefore, this study sought to achieve consensus for the test-treatment threshold for asthma diagnosis in adults; identifying the probability at which primary care clinicians can feel confident they have enough information to make a diagnosis, and information from additional investigations is unlikely to change their diagnosis.

\section{METHODS}

We undertook a consensus workshop using modified nominal group technique (NGT) methodology. ${ }^{17} 18$ The workshop was held during an International Primary Care Respiratory Group (IPCRG) conference in Bucharest, Romania, in May 2019. Workshop materials were developed by the research team and piloted during a practice event held with colleagues from the Asthma UK Centre for Applied Research, 6 weeks in advance.

\section{Recruitment}

Potential participants were identified from the publicly available IPCRG conference programme with support from the conference organisers. Participants were eligible if they had knowledge and experience of working in primary care or equivalent settings and had sufficient fluency in English to take part in the discussion. We sought 10 participants (the suggested number for a modified NGT consensus ${ }^{17}$ from clinical backgrounds with a specialist interest in asthma and from a range of countries. Potential participants were contacted by email with an information sheet.

\section{Data collection}

A first round of voting was conducted by email 2 weeks before the face-to-face workshop. Participants were asked, 'At what probability would you consider someone to be at high probability of asthma diagnosis?' and provided an answer (an integer between $0 \%$ and $100 \%$ ) anonymously.

At the beginning of the face-to-face workshop, participants were provided with a detailed explanation of the session and were asked to sign a consent form. Participants received a $20 \mathrm{~min}$ presentation with definitions of key terms, an explanation of the rationale and anonymised feedback from the first round of voting (table 1). Remaining uncertainties were addressed before participants voted for a second time (anonymously) on

Table 1 Outline of the information provided to participants before round 2 voting

\begin{tabular}{ll}
\hline Content & Description \\
\hline $\begin{array}{l}\text { Challenges } \\
\text { relating to } \\
\text { asthma } \\
\text { diagnosis }\end{array}$ & $\begin{array}{l}\text { Definition of asthma, rates and } \\
\text { consequences of misdiagnosis and the } \\
\text { lack of a 'gold' standard test for asthma } \\
\text { were presented. }\end{array}$ \\
$\begin{array}{l}\text { Approaches } \\
\text { to asthma } \\
\text { diagnosis }\end{array}$ & $\begin{array}{l}\text { Recommendations from the Global } \\
\text { Initiative for Asthma }{ }^{4} \text { and the National } \\
\text { Institute for Health and Care Excellence }\end{array}$ \\
$\begin{array}{l}\text { Threshold } \\
\text { approach to presented. } \\
\text { diagnosis }\end{array}$ & $\begin{array}{l}\text { The threshold approach to diagnosis was } \\
\text { explained with asthma-specific cases and } \\
\text { diagrams. }\end{array}$ \\
$\begin{array}{l}\text { Probability } \\
\text { of an asthma } \\
\text { diagnosis }\end{array}$ & $\begin{array}{l}\text { Two cases were presented demonstrating } \\
\text { how the probability of a diagnosis can }\end{array}$ \\
& $\begin{array}{l}\text { vary, depending on the information } \\
\text { available at different times and before and } \\
\text { after investigations. }\end{array}$ \\
\hline
\end{tabular}


the question 'At what probability would you consider someone to be at high probability of asthma diagnosis?'

Anonymised results of the second round of voting were displayed graphically and reviewed by the group. Participants discussed the question and considered the pros and cons of choosing too high or too low a value for the threshold. Discussion was facilitated by two researchers, both academic general practitioners, with materials including two clinical cases used to direct the conversation. A final round of anonymous voting on the research question was completed, marking the end of the workshop.

\section{Data analysis}

Each round of participant voting was visualised graphically and analysed using descriptive statistics (mean, SD and range) using $\mathrm{R}$. The workshop was, with permission of participants, audio-recorded and transcribed. The workshop discussion was analysed qualitatively using a thematic approach. ${ }^{19}$ The transcript was read, and initial coding was completed using NVivo V.10 (QSR International). Codes were collected into potential themes, and a final coding framework agreed on following discussion within the research team.

\section{RESULTS}

A total of 26 people were approached (selected to try and achieve a broad range of nationalities), of whom 10 participants ( 4 women) from nine countries were available and recruited into the study. Table 2 lists the countries of origin and the professional backgrounds of the participants. Though not formally assessed, each participant was sufficiently fluent in English to take an active role in the discussion. All participants had a specialist interest in respiratory healthcare. A complete set of votes was received for every round except for one participant in round 1. Based on the final round of voting, the mean consensus value for the threshold constituting high probability of asthma was 75\% (SD 7.6). By the final round of voting, there was less variation between participants

Table 2 Countries of origin and professional background of the participants

\begin{tabular}{lll}
\hline Region & Countries & Professional role \\
\hline Northern Europe & $\begin{array}{l}\text { Germany, } \\
\text { Netherlands, } \\
\text { Sweden, UK }\end{array}$ & $\begin{array}{l}\text { 4 Academic GPs } \\
\text { 1 GP with multiple } \\
\text { roles }\end{array}$ \\
$\begin{array}{lll}\text { Southern Europe } \\
\text { Eastern Europe }\end{array}$ & $\begin{array}{l}\text { Portugal, Spain } \\
\text { Romania }\end{array}$ & $\begin{array}{l}\text { Academic GPs } \\
\text { physician }\end{array}$ \\
$\begin{array}{l}\text { Far East } \\
\text { subcontinent }\end{array}$ & Malaysia & 1 Academic GP \\
Australasia & Australia & 1 Academic GP \\
\hline
\end{tabular}

GP, general practitioner.
Table 3 Participant votes across the three rounds

\begin{tabular}{lcll}
\hline Participant & Round 1 & Round 2 & Round 3 \\
\hline A & $80 \%$ & $75 \%$ & $70 \%$ \\
B & $95 \%$ & $95 \%$ & $65 \%$ \\
C & $80 \%$ & $70 \%$ & $65 \%$ \\
D & $70 \%$ & $90 \%$ & $80 \%$ \\
E & $80 \%$ & $80 \%$ & $65 \%$ \\
F & $80 \%$ & $70 \%$ & $80 \%$ \\
G & $80 \%$ & $85 \%$ & $85 \%$ \\
H & $100 \%$ & $80 \%$ & $80 \%$ \\
I & - & $90 \%$ & $80 \%$ \\
J & $80 \%$ & $65 \%$ & $75 \%$ \\
Mean (SD) & $83 \%(9.1)$ & $80 \%(10.0)$ & $75 \%(7.6)$ \\
\hline
\end{tabular}

compared with rounds 1 and 2, with the lowest and highest votes at $65 \%$ and $85 \%$, respectively (table 3 ).

Figure 1 displays how voting frequencies changed between rounds. The first- round, completed via email, showed a peak of votes at $80 \%$, with few participants choosing a 'high' threshold (eg, 95\% or 100\%). Round 2, completed after a 20 min introduction and explanation, demonstrated more variation among participants, with no clear majority. The final voting round, after the facilitated discussion, showed a general reduction in the threshold voted for and two values, $65 \%$ and $80 \%$, attracting several votes.

\section{Influences on participant decision making}

Reviewing the discussion identified a number of factors that shaped the evolution of participants' decision making on what the threshold should be. The context in which

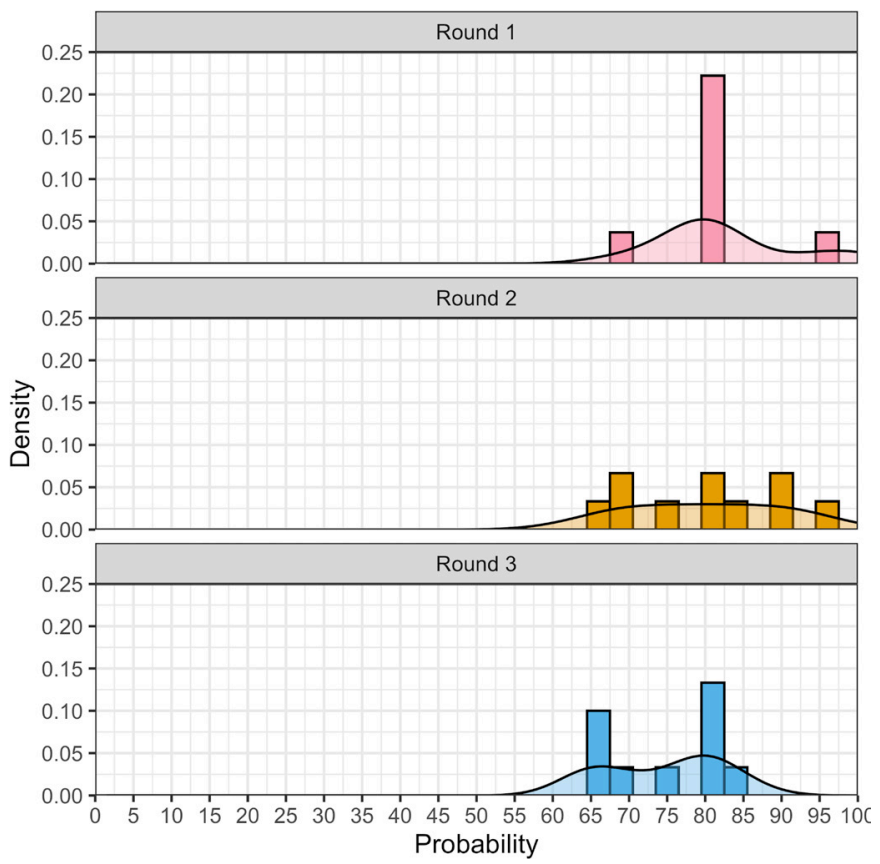

Figure 1 Histogram with density plot demonstrating the voting frequencies from each round. 
each participant worked and the resources available to them influenced their choice of threshold. Some participants had ready access to investigations, which enabled them to complete testing quickly and easily.

I have a spirometer in my office and [...] I love to depict the patients at once. Participant F

Another participant explained that the timing at which spirometry was performed could make a difference to the interpretation:

We can get a full spirometry in a week where I work. But if we would have to wait for 2 weeks then they [the patient] would probably be better by then. So, it depends on when they get the test. Participant H

In contrast, participant B shared how she dealt with a patient with suspected asthma in a low resource setting:

We don't have access to spirometry, and we have very limited peak flow at the clinics, so if I have a patient at the clinic presenting with wheezing and some degree of asthma history, the aim is to provide a treatment so that they can go home better rather than really getting the diagnosis. Participant B

\section{'Asthma' as a diagnostic label?}

Regardless of the health system or availability of investigations, some participants argued that providing treatment may be an appropriate option even without a precise diagnostic label:

Well it's all about the 'game' you are in... and the 'game' we are in is reducing the number of symptoms and reducing the risk, that's the 'game' we are in. We are not in the 'game' of this is asthma or this is not asthma. Participant J

...it's our job as a GP to decide if someone is ill or not together with the summarisation of different test(s), so if someone has a really clear history [of asthma] but spirometry doesn't help me in that patient at that moment, then I treat him despite a negative spirometry. Participant F

In addition, participant $\mathrm{C}$ explained that using asthma as a diagnostic label was a problem as contemporary understanding of asthma is not as a single disease:

...asthma is not a disease, there is not one asthma, there is different 'asthmas'...So that is the main problem, we are diagnosing with one word many diseases, different pathophysiologies, different things. Participant C

\section{Different 'tests' for asthma}

In the absence of a 'gold standard' investigation for asthma and understanding asthma to be a heterogeneous disease, participants discussed strategies they used to make a diagnosis of asthma:
We get information from several diagnostic tests. So, the medical history is a diagnostic test, physical examination is a diagnostic test, spirometry, peak flow variability and so on. And we as physicians sometimes decide which test we accept, or refuse, and we make a mixture in our minds somehow and come to the final diagnosis. Participant F

Response to treatment was also considered to be an important tool in weighing up the evolving likelihood of asthma:

I use that trial [of treatment] as one of my tests. I have a different probability depending on their response to that trial than I would making that decision. So, my threshold for instituting the trial of treatment might be 50 or $60 \%$ but my probability where no test is going to change my mind, after that trial would be quite different. Participant I

\section{Consequences of a low threshold for diagnosing asthma}

Discussing the likely clinical course when making a diagnosis of asthma helped some participants to consider the acceptability of diagnosing asthma at a lower probability:

'I'm not going to operate on them, I'm not going to give them radiotherapy or chemotherapy, I'm going to give them a bit of inhaled corticosteroid for sixweeks...my level of probability has actually come down a bit as I've begun to think about it because I'm not so worried about the consequence of having a lower threshold because it's not so bad having six weeks of inhaled corticosteroid.' Participant A

If a lower threshold for diagnosing asthma was used, more people would be labelled as having asthma, and if started on first-line treatment (low-dose inhaled corticosteroids), the risk of asthma attack would potentially be lower. Participant I highlighted how an episode of thunderstorm asthma demonstrated the potential for severe and fatal asthma attack even in those not aware of having the disease:

we had 11 people die, [and] a whole heap hadn't had or didn't think they had asthma, and these were the ones that ended up in the emergency department. Participant I

\section{Advantages of a high threshold for diagnosing asthma}

While there could be advantages for a lower threshold for diagnosing asthma, a consequence would be an increase in the number of patients wrongly labelled with asthma (false positives). Though the initial treatment of asthma may be considered relatively safe, providing ongoing treatment for the wrong diagnosis would have implications:

A person who hasn't got asthma, who is being treated for asthma with escalating doses of anti-inflammatory medication, either high dose inhaled corticosteroids or oral corticosteroids are being subject to a 
medication that isn't going to help them, but they are also not getting treatment for the disease that they have got. So, it's a double whammy. Participant G

If a person was wrongly labelled with asthma, the consequences would also depend on the missed diagnosis that was the cause for the symptoms:

There are no consequences of actually having the wrong diagnosis if the alternative is that she doesn't have any other diagnosis. But if the alternative is that she has a lung embolus, she's dead next day. Participant J

\section{Safety net of follow-up}

As well as considering consequences, participants discussed how confident they would be that a person who had been misdiagnosed would be identified and subsequently correctly diagnosed. In the case of someone starting treatment for asthma, participant D explained that in her context, once a person had been started on a treatment, there would be an opportunity to review the treatment and ultimately check that the diagnosis was correct:

The GP is obliged to send to a specialist to confirm the diagnosis, to review the treatment. I think that is a good thing because the patient comes to me 'just give me another letter to continue the treatment' and I say no, we have to review the diagnosis with spirometry at the clinic and step [treatment] up or down. Participant D

Participant E agreed that it 'might be very nice confirming all your [asthma] diagnoses' but felt it was 'unthinkable' in their country because it would [flood the hospitals]; therefore, review was organised within primary care. For participant B, reviewing patients was harder as they explained often '[people] won't come back'.

In the situation where a person did have asthma, but it had not been identified, participant $\mathrm{E}$ felt that '[the diagnosis] can only be recognised and corrected if the patient comes back with symptoms'.

\section{Sharing the uncertainty}

Participant A elaborated further by explaining that if asthma had been considered but decided against, discussing this uncertainty with a patient felt like 'an easy conversation to have':

I guess most health professionals have a conversation saying 'I don't think this is asthma, if I don't treat you, you may carry on having symptoms for a while but if you don't then it means it wasn't that'...'I won't treat you [just yet] because I want to see whether or not you get better on your own.' Participant A

Dealing with uncertainty and screening for red flags were felt to be common features of general practice:
We have to be able to deal with uncertainty, but also to know very well your red flags... TB, pulmonary embolism and so on. Those are the red flags that we need to have all the time when we think this patient might have asthma but might have something else. And that something else needs to be ruled out for sure and yet still you have to live with uncertainty. That's the rule in general practice. Participant E

\section{DISCUSSION \\ Principal findings}

The consensus threshold at which the diagnosis of asthma in primary care was considered high probability was $75 \%$. This figure is the mean value of the threshold score of 10 international primary care-based respiratory interested participants and indicates the level at which participants were comfortable to code an individual with suspected asthma and start a monitored trial of treatment. Taken together with the qualitative data, the value may suggest a compromise between limiting the number of false positives that would be generated by using too low a threshold, and on the other hand, being pragmatic and understanding that as a first-line treatment, low-dose inhaled corticosteroid does not carry high risks if a review can be ensured at which the response to treatment can be assessed and treatment dosages adjusted.

\section{Strengths and weaknesses}

We opted to use modified NGT methodology as we felt a detailed discussion would be best achieved through face-to-face discussion. ${ }^{1718}$ Subsequently, we recruited 10 participants, the suggested number for a modified NGT. ${ }^{17}$ We acknowledge that with a small sample, our statistical analysis should be interpreted with caution as it is possible that had the participants been different or discussed the topic on another occasion, the voting may have been different. Using a mixed-methods approach helped to balance our interpretation.

We recruited from IPCRG conference attendees and our sample reflected the majority of conference delegates: doctors working in primary care from around the world with experience and interest in respiratory medicine. As a purposive sample, we achieved views from a range of nations, but the findings may not be applicable to all countries. Despite our efforts, we failed to recruit any nurses to this study. However, unlike in the UK where nurses have a central role, the diagnosis and management of asthma internationally is largely doctor-led. We would have achieved a broader perspective by including secondary/tertiary care clinicians, but we chose not to because the focus of the study was asthma diagnosis in primary care, and health problems such as respiratory symptoms typically present differently between primary and secondary care settings. ${ }^{2021}$ 


\section{Interpretation}

\section{Pragmatic practice}

Seventy-five per cent was the consensus value for the testtreatment threshold based on 10 participants, and its utility and safety needs to be further investigated before adoption into practice. In the final round of voting, there remained a spread of opinion; three participants voted for $65 \%$ and four for $80 \%$. Taken together with the qualitative analysis, we felt that there were two broad groups of opinion. For those opting for a lower threshold, there were a number of reasons that being able to make a diagnosis of asthma at lower probability made sense. First, having a high probability of disease was less important than delivering the right treatment. Understanding asthma to be an umbrella term (ie, not a single disease) raised questions about the validity and value of using asthma as a diagnostic term. ${ }^{22}$ Instead of asthma, 'treatable traits' offer a more personalised approach to classification and management, ${ }^{23}$ and the priority is in delivering the right treatment to the individual. This practical approach to 'making the patient better' was shared by those in lowresource settings who lacked investigations and had little confidence that a patient would return for a review. The pragmatic approach was to exclude serious differential diagnoses (such as tuberculosis (TB) and pulmonary embolus) and to provide treatment for the most likely problem.

Those who chose $80 \%$ as a threshold for high probability sought greater confidence that what they were treating had a diagnostic label of asthma. Being able to access the right investigations at the right time was important and provided confidence that the right diagnosis could be achieved. Reducing unnecessary prescription of inhaled corticosteroids was also a priority, with concern that misdiagnosis can lead to escalation of treatment to high-dose therapies.

\section{Current guidelines}

Currently, there is no definitive evidence to recommend the best approach for diagnosing asthma in clinical settings, and consequently, international guidelines make different recommendations. ${ }^{412} 13$ The BTS/SIGN (2019) guideline recommends that those at high probability of asthma (defined as those having a clinical assessment 'typical' of asthma and no features to suggest an alternative diagnosis) should be recorded as suspected asthma and should begin a monitored trial of treatment. ${ }^{12}$ The recommendation is pragmatic, recognising that in some instances, when the clinical picture is overwhelmingly in favour of asthma, reviewing after a trial of treatment may be the most appropriate course. However, the concept of high probability may be interpreted differently among health professionals and can lead to treatment being started inappropriately rather than seeking to confirm the diagnosis with objective testing, principally spirometry with reversibility. ${ }^{2}$

Asthma is a variable condition and demonstration of objective improvement with treatment (bronchodilators, inhaled steroids and oral steroids) may contribute to the diagnostic assessment. The concept of a 'trial of treatment' has been criticised as potentially leading to commencement of lifelong treatment without a clear diagnosis. Most participants in this study took a different view, considering that commencing treatment as a formal 'trial' was the best way to prevent ongoing inappropriate prescribing. Indeed, it was the recognition that, as primary care clinicians they could ensure a review to assess response and titrate down (and potentially stop) treatment that contributed to a reduction in the testtreatment threshold for some participants. This echoes the structured approach to diagnosis illustrated in the BTS/SIGN (2019) guideline as a gap between a trial of treatment in a patient 'suspected' of having asthma and commencing regular medication. ${ }^{12}$ However, if a structured approach to diagnosis is not followed and individuals suspected of having asthma are not reassessed appropriately, poorly undertaken treatment trials may contribute to misdiagnosis, with individuals treated inappropriately and unnecessary medication costs to the healthcare system. ${ }^{24}$

In other guidelines, objective tests form a key part of the diagnostic workup. ${ }^{413}$ A lack of consistent objective testing (such as spirometry, peak flow and bronchial challenge testing) has been associated with a failure to confirm current asthma, ${ }^{2}$ leading to recommendations to shift from a perceived 'no-test culture' in asthma. ${ }^{22}$ In this study of participants from different international healthcare settings, the ability to access and perform tests when they were needed varied. Some participants had spirometry in their office, but most had to wait for a period before spirometry could be completed, and one participant had no access to lung function testing. Accordingly, each participant had developed strategies for diagnostic investigation in the context in which they worked.

\section{Ensuring follow-up}

In this study, the final value for the test-treatment threshold $(75 \%)$ was lower than the first two rounds of voting. Qualitative analysis of the discussion indicates that following the introductory presentation (before round 2), participants discussed the diagnostic process and were confident that, regardless of the probability threshold they chose, systems could be put in place to ensure patients were reassessed. Whether to correct a false-positive diagnosis and stop treatment or to remedy a false-negative diagnosis if symptoms developed, being able to review a patient was felt to be crucial in confirming (or changing) a diagnosis. Unfortunately, in practice, ensuring the review of individuals can be challenging, meaning that a diagnostic label or treatment response goes unreviewed. Using a suspected asthma code is one way in which patients in whom the diagnosis has not been established may be identified, making it clear to health professionals in the future that the working diagnosis remains unverified. ${ }^{12}$ 
Implications for policy makers, healthcare professionals and researchers

This study demonstrates that approaches for assessing asthma in primary care are strongly influenced by the clinical context in which they occur. However, there are general principles which policy makers should consider to help primary care clinicians improve the accuracy of an asthma diagnosis. Investigations for asthma, such as spirometry, should be accessible and achievable in a timely fashion. In low-resource settings, understanding how best to diagnose asthma with limited availability of investigations is important,${ }^{25}$ but with increasing technological solutions such as apps to allow lung function testing by mobile phone,${ }^{26}$ reliable methods to identify variable lung function should become more widespread. In high-resource settings, different healthcare models for achieving diagnostic tests exist, each with advantages and disadvantages. Respiratory testing within practices can be accessible for patients and potentially more straightforward to complete when a patient is symptomatic; however, practices have concerns about the time taken, ${ }^{27}$ and maintaining quality assurance can be resource intensive. Alternatives such as diagnostic referral centres, ${ }^{28}$ where a patient may attend for a wider range of tests in a single sitting, provide economies of scale but might lead to a deskilling of primary care practitioners. ${ }^{14}$

Ensuring follow-up of patients during the diagnostic period will require varied solutions across different health systems. In low-resource settings, TB-specific programmes have shown improvements in follow-up after outpatient visits, ${ }^{29}$ and plans to extend these initiatives to other diseases are being attempted as part of wider efforts to improve continuity of care and patient-centred universal health coverage. ${ }^{30}$ In high-resource settings, such as the UK, policy makers and health professionals should consider how follow-up may be affected as continuity in primary care becomes harder to achieve. ${ }^{31}$

Findings from this study will inform the development of a clinical decision support system (CDSS) to aid the diagnostic decision making of asthma by primary care health professionals. In addition to providing a structured approach to diagnosis, a CDSS could also provide strategies to deal with diagnostic uncertainty. Shared decision making is often considered in the context of screening and treatment decisions. ${ }^{32}$ Yet, uncertainty may be greater in diagnostic situations as, unlike treatment decisions where an existing condition has been categorised, diagnosis requires clarification of the cause of symptoms, making it more difficult to weigh up the options and to decide on a management course. ${ }^{32}$ CDSS and computer screen sharing during a consultation may offer practical solutions; for example, a structured approach guided by a CDSS embedded in the practice computer may change organisational pathways; sharing diagnostic uncertainty with the patient may help in communicating the importance of a review and improve the chances of follow-up. ${ }^{3334}$

\section{CONCLUSION}

A probability of $75 \%$ was the consensus threshold at which participants in this workshop felt confident to establish the diagnosis of asthma in primary care, although with the caveat that a review of treatment response was essential. Contextual factors, including availability and timing of tests and the ease with which patients could be reviewed, also influenced participants' decision making.

\section{Twitter Luke Daines @ljdaines}

Acknowledgements We are grateful to the International Primary Care Respiratory Group for facilitating the research meeting, to Adeola Akindele for workshop support, to Holly Tibble for help with $\mathrm{R}$ and all the workshop participants for taking part.

Contributors HP, LD and ASc conceived the idea for this work, supported by ASh and SL. All authors contributed to the interpretation of the findings. The paper was drafted by LD and HP and revised after feedback from all other authors. All authors have seen and approved the final version.

Funding This work was supported by the Chief Scientist Office (grant number CAF/17/01).

Competing interests LD and HP are on the British Thoracic Society (BTS)/Scottish Intercollegiate Guideline Network (SIGN) asthma guideline development committee. ASh has previously served on the BTS/SIGN Asthma Guideline Committee and currently serves on Global Initiative for Asthma's Scientific Committee. ASc is a member of the Committee of the German National Asthma Guideline (Nationale VersorgungsLeitlinie Asthma (NVL) Asthma).

Patient and public involvement The programme of research benefits from the involvement of users, both patients and professionals, who have provided feedback on the study design, informational material and acted as co-applicants on grants and members of the project steering group.

Patient consent for publication Not required.

Ethics approval The study protocol was reviewed and approved by the University of Edinburgh sponsor (number: AC19047), which recommended that no further ethical review was required.

Provenance and peer review Not commissioned; externally peer reviewed.

Data availability statement No data are available. We do not have consent to share the primary data collected with other researchers.

Open access This is an open access article distributed in accordance with the Creative Commons Attribution Non Commercial (CC BY-NC 4.0) license, which permits others to distribute, remix, adapt, build upon this work non-commercially, and license their derivative works on different terms, provided the original work is properly cited, appropriate credit is given, any changes made indicated, and the use is non-commercial. See: http://creativecommons.org/licenses/by-nc/4.0/.

ORCID iD

Luke Daines http://orcid.org/0000-0003-0564-4000

\section{REFERENCES}

1 van Schayck CP, Chavannes NH. Detection of asthma and chronic obstructive pulmonary disease in primary care. Eur Respir J Suppl 2003;39:16S-22.

2 Aaron SD, Vandemheen KL, FitzGerald JM, et al. Reevaluation of diagnosis in adults with physician-diagnosed asthma. JAMA 2017;317:269-79.

3 Looijmans-van den Akker I, van Luijn K, Verheij T. Overdiagnosis of asthma in children in primary care: a retrospective analysis. $\mathrm{Br} \mathrm{J} G e n$ Pract 2016;66:e152-7.

4 Global Initiative for Asthma. Global strategy for asthma managment and prevention, 2019. Available: www.ginasthma.org

5 Osborne ML, Pedula KL, O'Hollaren M, et al. Assessing future need for acute care in adult asthmatics: the profile of asthma risk study: a prospective health maintenance organization-based study. Chest 2007;132:1151-61.

6 Schneider A, Gindner L, Tilemann L, et al. Diagnostic accuracy of spirometry in primary care. BMC Pulm Med 2009;9:31. 
7 Hunter CJ, Brightling CE, Woltmann G, et al. A comparison of the validity of different diagnostic tests in adults with asthma. Chest 2002;121:1051-7.

8 Crapo RO, Casaburi R, Coates AL, et al. Guidelines for methacholine and exercise challenge testing-1999. this official statement of the American thoracic Society was adopted by the ats board of directors, July 1999. Am J Respir Crit Care Med 2000;161:309-29.

9 Karrasch S, Linde K, Rücker G, et al. Accuracy of FENO for diagnosing asthma: a systematic review. Thorax 2017;72:109-16.

10 den Otter JJ, Reijnen GM, van den Bosch WJ, et al. Testing bronchial hyper-responsiveness: provocation or peak expiratory flow variability? Br J Gen Pract 1997;47:487-92.

11 Tilemann L, Gindner L, Meyer FJ, et al. [Diagnostic value of peak flow variability in patients with suspected diagnosis of bronchial asthma in general practice]. Dtsch Med Wochenschr 2009;134:2053-8.

12 Health Improvement Scotland. British guideline on the management of asthma, 2019. Available: https://www.sign.ac.uk/assets/sign158. pdf

13 The National Institute for Health and Care Excellence. Asthma: diagnosis, monitoring and chronic asthma management, NICE nG80, 2017. Available: https://www.nice.org.uk/guidance/ng80

14 Keeley D, Baxter N. Conflicting asthma guidelines cause confusion in primary care. BMJ 2018;360:k29-10.

15 Pauker SG, Kassirer JP. The threshold approach to clinical decision making. N Engl J Med 1980;302:1109-17.

16 Daines L, McLean S, Buelo A, et al. Systematic review of clinical prediction models to support the diagnosis of asthma in primary care. NPJ Prim Care Respir Med 2019;29:1-4

17 Murphy MK, Black NA, Lamping DL, et al. Consensus development methods, and their use in clinical Guideline development. Health Technol Assess 1998;2:i-iv, 1-88.

18 Bernstein SJ, Laouri M, Hilborne LH, et al. Coronary angiography: a literature review and ratings of appropriateness and necessity. Santa Monica (CA), 1992.

19 Braun V, Clarke V. Using thematic analysis in psychology. Qual Res Psychol 2006;3:77-101.

20 Schneider A, Ay M, Faderl B, et al. Diagnostic accuracy of clinical symptoms in obstructive airway diseases varied within different health care sectors. J Clin Epidemiol 2012;65:846-54.

21 Knottnerus JA. Between iatrotropic stimulus and interiatric referral. $J$ Clin Epidemiol 2002;55:1201-6.
22 Pavord ID, Beasley R, Agusti A, et al. After asthma: redefining airways diseases. Lancet 2018;391:350-400.

23 Agusti A, Bel E, Thomas M, et al. Treatable traits: toward precision medicine of chronic airway diseases. Eur Respir J 2016;47:410-9.

24 The National Institute for Health and Care Excellence. Asthma: diagnosis and monitoring of asthma in adults, children and young people: appendices A-R, 2017. Available: https://www.nice.org.uk/ guidance/ng80

25 Pinnock H, Østrem A, Rodriguez MR, et al. Prioritising the respiratory research needs of primary care: the International primary care respiratory group (IPCRG) e-Delphi exercise. Prim Care Respir J 2012;21:19-27.

26 Larson EC, Goel M, Boriello G, et al. SpiroSmart. In: Proceedings of the 2012 ACM Conference on Ubiquitous Computing - UbiComp '12. 280. New York, New York, USA: ACM Press, 2012.

27 Akindele A, Daines L, Cavers D, et al. Qualitative study of practices and challenges when making a diagnosis of asthma in primary care. NPJ Prim Care Respir Med 2019;29:27.

28 Metting El, Riemersma RA, Kocks JH, et al. Feasibility and effectiveness of an asthma/COPD service for primary care: a crosssectional baseline description and longitudinal results. NPJ Prim Care Respir Med 2015;25:1-7.

29 Law S, Daftary A, O'Donnell M, et al. Interventions to improve retention-in-care and treatment adherence among patients with drug-resistant tuberculosis: a systematic review. Eur Respir $J$ 2019;53:1801030.

30 Schwarz D, Hirschhorn LR, Kim J-H, et al. Continuity in primary care: a critical but neglected component for achieving high-quality universal health coverage. BMJ Glob Health 2019;4:e001435.

31 Rosen R, Kumpunen S, Curry N. Is bigger better? Lessons for general practice, 2016.

32 Berger ZD, Brito JP, Ospina NS, et al. Patient centred diagnosis: sharing diagnostic decisions with patients in clinical practice. BMJ 2017;359:j4218.

33 Milne H, Huby G, Buckingham S, et al. Does sharing the electronic health record in the consultation enhance patient involvement? A mixed-methods study using multichannel video recording and indepth interviews in primary care. Health Expect 2016;19:602-16.

34 White A, Danis M. Enhancing patient-centered communication and collaboration by using the electronic health record in the examination room. JAMA 2013;309:2327. 